\title{
Aportes de los Nuevos Estudios de Literacidad AL CAMPO DE LA EDUCACIÓN DE JÓVENES Y ADULTOS
}

\author{
Contribuições dos Novos Estudos de Letramento aO CAMPO da EdUCAÇão \\ DE JOVENS E ADULTOS \\ Contributions of the New Literacy Studies to the Field of Youth and Adult \\ EDucation
}

\begin{abstract}
María del Carmen Lorenzatti Doctora en Ciencias de la Educación, Universidad Nacional de Córdoba, Argentina. Profesora adjunta en el área de Educación, Facultad de Filosofía y Humanidades, Universidad Nacional de Córdoba. Miembro grupo de investigación sobre educación de jóvenes y adultos, Centro de Investigaciones Facultad de Filosofía y Humanidades. Miembro del Consejo Internacional de Educación de Adultos (ICAE) y de la Cátedra Pablo Latapí, Argentina.

Pedro Echagüe 3092, Barrio San Fernando cp 5016, Córdoba, Argentina marial@ffyh.unc.edu.ar
\end{abstract}

\section{Gladys Blazich}

Doctoranda en Ciencias de la Educación. Especialista en formador de formadores (CREFAL) y en Gestión y conducción del sistema educativo y sus instituciones (FLACSO). Subdirectora del Instituto de Ciencias de la Educación, Facultad de Humanidades, Universidad Nacional del Nordeste, Argentina. Miembro grupo de investigación sobre Educación de jóvenes y adultos, Centro de Investigaciones, Facultad de Filosofía y Humanidades de la Universidad Nacional de Córdoba.

\section{RESUMEN}

En este artículo se exponen algunos aportes de la perspectiva de los nuevos estudios de literacidad (NEL) al campo de la educación de jóvenes y adultos. Esta perspectiva y el uso del enfoque etnográfico posibilitan el registro, el análisis y la conceptualización de las prácticas cotidianas de los sujetos en relación con la cultura escrita. En esta línea se exponen los hallazgos de dos investigaciones realizadas en escuelas de jóvenes y adultos en Argentina. En el primer apartado se expone el abordaje teóricometodológico de los NEL. Posteriormente, se analizan prácticas de lectura y escritura a partir de los usos y sentidos asignados al abecedario. En el segundo apartado se puede observar cómo a partir de la lectura de un cuento se configuran las relaciones con la cultura escrita en la escuela. Finalmente se presentan algunas reflexiones sobre el aporte de los NEL a la educación de jóvenes y adultos en Argentina.

Palabras claves: jóvenes y adultos; educación de jóvenes y adultos; literacidad; escuelas de jóvenes y adultos.

\section{Resumo}

No artigo apresentam-se as contribuições que a perspectiva dos novos estudos de letramento (NEL) realiza ao campo da educação de jovens e adultos. Esta perspectiva desenvolve-se em conjunto com o enfoque etnográfico que possibilita o registro, a análise e a conceptualização das práticas cotidianas dos sujeitos com relação à cultura escrita. Nesta linha expõe-se as descobertas de duas pesquisas realizadas em escolas de jovens e adultos na Argentina. Na primeira parte apresenta-se a abordagem teóricametodológica proposta pelos NEL. Após, se analisam práticas de leitura e escritura a partir dos usos e sentidos dados ao abecedário escolar. Na seguinte parte visibiliza-se como se configuram as relaçóes com a cultura escrita na escola a partir da leitura de um conto. Finalmente se apresentam algumas reflexões sobre a contribuição dos NEL à educação de jovens e adultos na Argentina.

Palavras-chave: jovens e adultos; educação de jovens e adultos; letramento; escolas de jovens e adultos.

Recibido: 2018-07-23/ Aceptado: 2018-11-08 / Publicado: 2019-05-01 
Miembro de la Cátedra Pablo Latapí, Argentina.

Las Heras 727-3500 Resistencia, Chaco gblazich@yahoo.com.ar

\section{Rocío Arrieta}

Licenciada en Ciencias de la Educación, Escuela de Ciencias de la Educación, y doctoranda en Ciencias de la Educación, Facultad de Filosofía y Humanidades, UNC. Becaria de Postgrado (2012-2017), IDH-CONICET (Instituto de Humanidades del Consejo Nacional de Investigaciones Científicas) Córdoba, Argentina.

Miembro grupo de investigación sobre Educación de jóvenes y adultos, Centro de Investigaciones, Facultad de Filosofía y Humanidades de la Universidad Nacional de Córdoba.

Pedro Echagüe 3092, Barrio San Fernando, CP 5016 , Córdoba, Argentina. rocioaz@hotmail.com

\begin{abstract}
This article deals with the contributions brought by the New Literacy Studies to the field of youth and adult education. This perspective and the ethnographic approach allow to record, analyse and conceptualize individuals' everyday activities concerning written culture. Thus, the findings from two empirical research projects carried out in Argentina are presented here. In the first section, the methodological theoretical approach by NELs is presented. Then, reading and writing practices are analyzed from the uses and meanings assigned to the school's alphabet. In the following section it is possible to visualize how the relationships with the written culture in the school are configured from the reading of a story. Finally some reflections on the contribution of the NEL to the Education of Young People and Adults in Argentina are presented.
\end{abstract}

Keywords: young people and adults; education of young people and adults; literacy; schools for young people and adults.
Proyecto de investigación: "Prácticas educativas con jóvenes y adultos: políticas, sujetos y conocimiento", con lugar de trabajo en el Área Educación del Centro de Investigaciones de la FFyH. Aprobado y financiado por FONCYT. PICT-2016-2081. Res. No 285/2017. 


\section{Introducción}

La alfabetización de adultos ha sido un tema de debate de distintas políticas educativas - nacionales e internacionales-, de investigadores, de organizaciones sociales y de maestros preocupados por este tema. En nuestras aulas no estuvimos ajenos a esta preocupación. En el inicio de las clases del seminario de "Alfabetización y educación básica de jóvenes y adultos", durante mucho tiempo hicimos claridad sobre el sentido que damos a la palabra alfabetización. Sentíamos la necesidad de incluir la dimensión social de este proceso, de estudiar los sentidos que los sujetos confieren a la lectura y la escritura.

Con la inclusión de diferentes perspectivas teóricas fuimos construyendo una nueva forma de abordar los procesos de alfabetización. En primer lugar, la lectura de Freire (1971) nos llevó a reconocer la diferencia entre la educación bancaria y la educación como práctica de la libertad. Paralelamente, desde la psicogénesis, Ferreiro y Teberosky (1980) nos brindaron herramientas para pensar que, si los niños construyen hipótesis en torno a la cultura escrita, también lo harían los jóvenes y adultos. Sin embargo, no encontrábamos puentes para cruzar hacia la práctica de los maestros. A la hora de trabajar con ellos y con alfabetizadores, las propuestas de enseñanza mantenían el sesgo de la decodificación del fonema en grafema, de la repetición mecánica de sílabas. En los talleres realizados con los maestros identificamos sus sinsabores y angustias unidos a una sensación de fracaso producida por la mirada de la propia práctica. En nuestras investigaciones observamos que la misma no habilita ni promueve procesos de apropiación de la cultura escrita en personas que hace tiempo dejaron la escuela o nunca pudieron ingresar a ella.

1 Seminario que se dicta en la Escuela de Ciencias de la Educación, Facultad de Filosofía y Humanidades, Universidad Nacional de Córdoba, desde el año 1997. Actualmente se denomina Procesos de literacidad y educación básica de jóvenes y adultos.
El acceso a materiales bibliográficos sobre la perspectiva sociocultural y los nuevos estudios de literacidad (Street, 1993, 2005; Kalman, 2003, 2004; Zavala, 2002, entre otros) nos permitió mirar de otra manera los procesos de lectura y escritura. Este abordaje teórico metodológico nos abrió la posibilidad de articular estos procesos al concepto de "vida cotidiana" (Heller, 1977). También recuperamos el enfoque etnográfico en el trabajo, en la medida en que propicia el registro y análisis de las prácticas cotidianas de los sujetos en relación con la cultura escrita.

La pregunta que orienta el presente artículo es cuáles son los aportes de la perspectiva de los nuevos estudios de literacidad (en adelante, NEL) a la comprensión de las prácticas de lectura y escritura en escuelas para jóvenes y adultos. En primer lugar, presentaremos una aproximación conceptual de los NEL, examinando las herramientas metodológicas desplegadas en sus investigaciones; en segundo lugar, compartiremos hallazgos de dos proyectos de investigación, ${ }^{2}$ que forman parte de un proyecto global, ${ }^{3}$ en el campo de la educación de jóvenes y adultos. Finalmente realizaremos apreciaciones conceptuales derivadas de discusiones entre los miembros del equipo, que enriquecen y arrojan nuevas luces sobre los estudios de lectura y escritura en este campo socioeducativo.

\section{Abordaje teórico-metodológico}

En el marco de los NEL, se entiende la lectura y la escritura como prácticas inherentes a las relaciones sociales, y que no pueden reducirse a técnicas y habilidades individuales. Por lo tanto, es necesario comprender las prácticas letradas como

2 Cfr. Arrieta (en preparación) y Blazich (en preparación).

3 El equipo de investigación es dirigido por María del Carmen Lorenzatti, con sede en el Área Educación del Centro de Investigaciones de la Facultad de Filosofía y Humanidades, Universidad Nacional de Córdoba. El proyecto global "Prácticas educativas con jóvenes y adultos. Políticas, sujetos, conocimientos" está evaluado y financiado por SECYT (UNC- Período 2016-2017) y FONCYT (Período 2017-2020-ANPCYT) 
"íntegramente conectadas al mundo social, a partir de determinadas formas de actuar y de creer, y de diversas maneras de interactuar con el lenguaje oral y de utilizar herramientas y tecnologías" (Zavala, Murcia y Ames, 2004, p. 9). En este sentido Kalman (2002) sostiene que ser lector y escritor se desarrolla sobre una historia social en la cual se construye y se consolida una configuración de opciones culturales alrededor de la lengua escrita y una historia individual de nuestro propio tránsito por la geografía comunicativa.

Street (1984, citado en Street, 2008), desde los NEL, diferencia entre un modelo de alfabetización "autónoma” y un modelo de alfabetización "ideológica". El primero hace referencia a una práctica que se presenta como neutral y universal con efectos directos sobre otras prácticas sociales y cognitivas.

El modelo alternativo, ideológico, de la alfabetización brinda un punto de vista culturalmente sensible respecto a las prácticas alfabetizadoras, conforme varían de un contexto a otro. [...] la alfabetización constituye una práctica social y no únicamente una habilidad técnica y neutral; además sostiene que siempre está inmersa en principios epistemológicos socialmente construidos. (Street, 2008, p. 44)

En esta línea, Zavala et al. (2004, 2002) consideran que es necesario distinguir entre alfabetización y literacidad. Mientras la primera limita su significado al aspecto mecánico y técnico de codificación y decodificación de símbolos gráficos en el ámbito educativo - un concepto tecnicista, ligado a la educación formal- la literacidad hace referencia a la práctica social de lo letrado en cualquier contexto sociocultural, es decir, "la literacidad no constituye un bien en sí mismo sino una práctica social que está inserta en nuestro habitus cotidiano y, por consiguiente, no es la tecnología, sino las prácticas sociales y culturales relacionadas con ellas las que pueden beneficiar o poner en desventaja al usuario" (Zavala, 2002, p. 97). La autora considera que los NEL se constituyen a partir de una mirada interdisciplinaria que entiende lo letrado como un sistema simbólico arraigado en la práctica social. Street (1993) señala que la pluralidad de lo letrado es una construcción histórica y cultural y, por ende, inserta en relaciones de poder.

En América Latina, no existe una traducción lineal del concepto de literacy. Autores como Zavala, Niño, Murcia y Ames (2004) proponen como traducción el término literacidad y sus variaciones literacidades y biliteracidades (Kalman y Street 2009). A su vez, en Brasil se conoce a esta perspectiva teórica como letramento. La investigadora brasilera Tfouni considera que es un término polisémico que designa a "ese proceso de estar expuesto a los usos sociales de la escritura, sin saber leer ni escribir" (Tfouni, 2010, p. 10). Kalman (1993, citada en Kalman y Street, 2009, p. 10) señala que la palabra alfabetización no alcanza para designar fenómenos que van más allá de los aspectos más concretos de lectura y escritura y para ello reconoce la búsqueda de frases explicativas tales como "el uso de la lengua escrita", "el complejo fenómeno de la escritura" o "el aspecto social de la lengua escrita". En esa línea entiende que es difícil la traducción de literacy al español. La autora reconoce que el vocablo alfabetización remite a atributos negativos, como es el analfabetismo o analfabeto, que imprimen un sello estigmatizante asociado a la posición social y al nivel educativo alcanzado (Kalman, 2008).

En un trabajo reciente Knobel y Kalman (2017) hablan de prácticas del lenguaje porque hace referencia a lo oral, lo escrito y lo multimodal. Sostienen las autoras que se trata de "usos y comportamientos culturales reiterados, y da cuenta del uso competente de tecnologías diversas y de conocimientos, creencias y valorizaciones sociales" (p. 15).

En nuestras investigaciones realizamos una articulación conceptual entre la perspectiva de los NEL con la perspectiva sociológica de Heller (1977) y el concepto de vida cotidiana porque entendemos que contribuye a mirar a los sujetos desde sus modos cotidianos de apropiarse de aquellos aspectos de la cultura que se encuentran efectivamente en su ambiente inmediato, en su pequeño mundo, entre

4 La traducción es de las autoras de este artículo. 
ellos el lenguaje. Se incorpora así otro concepto relevante que es el de apropiación. Heller entiende que se trata de un proceso continuo y se centra en una relación activa entre las personas y la multiplicidad de recursos y usos culturales objetivados en distintos ambientes. Rockwell (2005, 2009) profundiza el análisis y sostiene que los procesos de apropiación se entienden en términos sociales, porque no se centran en el proceso cognoscitivo de aprendizaje que define la actividad del sujeto, sino en las relaciones y prácticas sociales cotidianas, a través de las cuales los sujetos se encuentran con otros y hacen suyos los conocimientos que construyen.

El abordaje teórico presentado necesariamente remite a un planteo metodológico, y de manera particular al enfoque etnográfico. Este es un punto nodal para pensar en la perspectiva de los NEL, porque el análisis de las prácticas en estudios desarrollados en diferentes contextos sociales e institucionales nos permite identificar de qué manera las políticas y los contextos interpelan a los sujetos y a la vez van configurando y forman parte de las prácticas; también orienta a desentrañar las acciones, los propósitos, los sentidos puestos en juego en las prácticas sociales de los sujetos y la manera como intervienen los conocimientos de los sujetos sobre la cultura escrita; como se incorporan los usos sociales de la lengua escrita al espacio educativo y en los momentos y las circunstancias en que los sujetos de los estudios realizados hacen uso de esos conocimientos fuera del espacio escolar. Este abordaje teórico metodológico permite reconocer las relaciones de poder donde se desarrollan las prácticas sociales en torno a la cultura escrita.

El "estar ahí", "mirar lo que sucede", "documentar lo no documentado” (Rockwell, 2009) implica también poner en marcha diferentes estrategias de obtención y análisis de la información. En relación con los estudios desarrollados en las escuelas de jóvenes y adultos, tienen prioridad las observaciones de clases y entrevistas a distintos sujetos (estudiantes, profesores, maestros y equipos directivos). En el caso de investigaciones que incorporan la mirada de lo que hacen los jóvenes y adultos con la cultura escrita fuera de la escuela, hemos desarrollado una manera de estar en el territorio que llamamos "acompañamiento" a los sujetos involucrados en los estudios. Esta estrategia posibilitó una mirada a las prácticas de literacidad en distintos espacios sociales con el objetivo de descentrar la mirada de la escuela como lugar privilegiado para el estudio de las prácticas de producción y uso de los conocimientos escolares - particularmente de las prácticas de lectura y escritura- y comprender cómo las experiencias vitales de los sujetos, ligadas a la cuestión laboral y las responsabilidades familiares se entraman en estos procesos.

En este artículo pretendemos compartir hallazgos de dos estudios que muestran el potencial analítico de la perspectiva de los Nel. Ambas investigaciones se desarrollan en escuelas de jóvenes y adultos de nivel primario de diferentes provincias de la Argentina y se intenta compartir el análisis sobre los modos como se incorpora la lengua escrita.

\section{El abecedario en la escuela. Usos y desusos.}

Es habitual asociar escuela y escritura. Esta asociación tiene como corolario considerar la escritura como un eje central para acceder a los conocimientos que la escuela transmite y superar los distintos niveles educativos. Sin embargo, enseñar a leer y escribir no fue siempre una misión exclusiva de la escuela. Kalman (2017), recuperando el trabajo de Cook-Gumperz (1988), analiza las transformaciones en los modos de transmisión de la cultura escrita a partir de la constitución de la escuela como modelo educativo hegemónico. Reconoce que, al inicio del siglo XIX, la lectura se aprendía por transmisión de otros lectores (padres, vecinos) en situaciones cotidianas, de manera similar a la transmisión de un oficio.

La escuela como institución pública avanzó sobre estas otras formas de diseminación y apropiación de la escritura y redujo la noción de alfabetización en cuanto a usos, procedimientos y propósitos de la lengua escrita. Así, la escritura en la escuela se 
constituyó en un fin en sí mismo, ajeno a otras actividades (Rockwell, 1997).

Kalman (2017) sostiene que la forma de leer y escribir en la escuela se identificó fundamentalmente con dos aspectos: el aprendizaje de formas gráficas con sus sonidos correspondientes (letras, sílabas y sonidos) y el reconocimiento de textos cortos sobre temas religiosos y morales (oraciones, fábulas con moralejas, entre otras). La autora sostiene que a pesar de los avances en la investigación, que dan cuenta de los distintos modos de relación de los sujetos con la escritura, la tendencia sigue siendo la enseñanza de la escritura a partir de elementos mínimos, como las letras y las sílabas. En los trabajos de investigación que realizamos, la escritura escolar se asocia además a la escritura sin errores ortográficos, a la prolijidad y a la "linda letra", aspectos que demandan un gran tiempo en la escuela (Lorenzatti y Arrieta, 2017). La escritura adquiere así características particulares tanto por los modos de transmisión de ese saber, como por las formas en que se prepara el espacio escolar para tal fin.

En este apartado analizamos distintos episodios que reflejan los usos del abecedario. Las observaciones se realizaron en un Centro Educativo de Jóvenes y Adultos de nivel primario (CEJA) de la ciudad de Córdoba, durante el período 2015-2016. El grupo de estudiantes estaba conformado por dos hombres y ocho mujeres, con edades entre los 18 y los 55 años, quienes asistían regularmente a la institución. Todos tenían trabajos informales y las mujeres eran madres de familias numerosas; algunas vivían con sus parejas y otras únicamente con sus hijos e hijas.

5 En Córdoba coexisten tres tipos de instituciones educativas para jóvenes y adultos de nivel primario con distintos orígenes y organizaciones institucionales. Los centros educativos de nivel primario de adultos (CENPA) y las escuelas nocturnas, ambos gestionados por el estado provincial y los centros educativos de jóvenes y adultos (CEJA) dependientes de las intendencias municipales. Los CEJA surgieron en el año 2001 en el marco de la fuerte crisis social y económica de nuestro país y se ubican en zonas periféricas de la ciudad.
La escuela de adultos funcionaba en el horario de 15 a 18 horas en un espacio en el que por la mañana atendía la secretaría de la escuela primaria regular (de niños). En este espacio convivían los muebles de la secretaría (un teléfono, una computadora, un armario y un escritorio) con el mobiliario escolar. Se observaba un pizarrón al frente, bancos separados y en fila y carteles que ornamentaban el aula o salón de clases.

En este pequeño espacio que la docente ornamenta como "aula" se observan distintos objetos escritos: un cartel con los nombres y las fechas de cumpleaños de los estudiantes, un mapa de la Argentina, una lista (ordenada alfabéticamente) con los estudiantes que asisten regularmente a la escuela y tres carteles (que se destacan por su tamaño y colorido) con el abecedario alfabético. Dos de ellos están colgados en ambas paredes del aula y el restante se encuentran al frente del grupo de estudiantes, abajo del pizarrón. Los abecedarios contienen además un dibujo en cada letra y una palabra que comienza con la letra correspondiente. Por ejemplo, en la letra $\mathrm{M}$ (el dibujo es un mono y la palabra escrita es mono). Este tipo de carteles son los que predominan en las escuelas primarias regulares.

Para Sanjuán (2015) el abecedario es un artefacto cultural del siglo XIX, cuya función esencial es enseñar a los niños a leer y escribir, "para reforzar la entidad gráfica y fonética de cada letra y facilitar el recuerdo, casi todos los abecedarios han seguido un esquema básico: la letra destacada se asocia con una imagen, generalmente de un objeto o animal, cuyo nombre empieza con dicha letra, y con una palabra o frase o una estrofa rimada en la que abunde esa letra [...]. En los abecedarios más simples se da un paralelismo estricto entre imagen y palabra" (Sanjuán, 2015, pp. 43 y 44). La autora reconstruye la historia del abecedario como artefacto estético y cultural. Sostiene que en principio el carácter didáctico de los abecedarios temáticos se dirigió hacia contenidos religiosos o morales. En el siglo Xx se modificaron, los abecedarios se hicieron más complejos en algunos casos, de manera que, según la autora, parecían destinados a lectores avanzados. 
En el aula observada, el abecedario no solo se constituye en un modo de ornamentación, sino en un recurso al que los estudiantes y la maestra apelan en el marco de la escuela para la concreción de actividades. Así, ante actividades como "completar la letra que falta en una palabra" o "colorear determinada letra en una palabra", la maestra solicita que miren el abecedario en caso de que se "olviden" la letra faltante o que no sepan la letra que deben colorear. Reconocemos cómo en el repertorio didáctico de la escuela predomina no solo la enseñanza del código - letra por letra, sílaba por sílaba- (Kalman, 2017), sino cómo las prácticas de escritura escolar y los artefactos culturales utilizados abonan a la construcción de significaciones particulares sobre la lengua escrita.

Para los estudiantes adultos aprender el abecedario es considerado un conocimiento fundamental en el proceso de apropiación de la escritura. En una de las observaciones en el aula, la maestra le indica a Sandra ${ }^{6}$ - una de las estudiantes del grupoque escriba su nombre en el cuaderno. Sandra lo hace con letra de imprenta y en mayúscula (mientras deletrea en voz baja). Una vez que termina, para controlar que lo hace correctamente, busca en su cuaderno dónde lo tiene escrito. Corrobora que su escrito esté bien y dice:

lo que pasa es que yo me olvido. Yo vine el año pasado también, pero me olvido, dejé a mitad de año, pero es así, me olvido y me olvido [...]. Mi marido me dice 'pero vos te tenés que concentrar' y es que a mí me gusta la charla, entonces yo me distraigo mucho. Yo me sé las letras, pero no las puedo poner juntas y mi marido me dice 'pero si vos sabes el abecedario ya está... si sabés las letras, solo le tenés que poner atención' (Sandra, Córdoba, 15/07/2015)

Para confirmar sus palabras y las de su marido, Sandra saca de su cuaderno dos abecedarios y los muestra - un abecedario tiene solo las letras y

6 Los nombres propios fueron modificados a fin de resguardar la identidad de los sujetos observados, tal como se acordó en el inicio de la investigación. el otro contiene las letras, acompañadas de una palabra y un dibujo, tal como el que está colgado en el aula-. Pasando el dedo sobre el abecedario, dice "lo que yo quiero es así leer rapidito, así, así rapidito".

Si bien Sandra dispone de distintos materiales escritos, como folletos de ofertas de supermercados, estampitas religiosas que comparte con sus compañeros de grupo, ella en distintas ocasiones deletrea el abecedario como un modo de demostrar su conocimiento de la cultura escrita. Estos haceres muestran que "no sólo sancionamos todas estas prácticas sociales mediante la interacción personal, humana, sino también al recurrir a objetos que nosotros, u otras personas, colocaron ahí para ayudar a estabilizar y asegurar el tipo de práctica social que se requiere" (Street, 2008, p. 51).

El abecedario se constituye en un artefacto que regula para Sandra la relación con la escritura en el ámbito escolar. El modo de hacerlo fortalece una idea de la escritura aislada y descontextualizada que se muestra ajena a otros contextos sociales y a otras actividades sociales extraescolares. Street (2008), citando los aportes de Bartlett y Holland, considera los artefactos como medios para representar quiénes somos y lo que está aconteciendo. En este sentido, Street expresa que

\section{El trabajo del salón de clases, el establecimiento de tipos especiales de relaciones sociales entre los partici- pantes (el papel del maestro, las suposiciones respecto al hecho de ser un aprendiz, los derechos determi- nados in situ de hablar y escribir) se llevan a cabo en parte gracias a los artefactos. (Street, 2008, p. 50)}

En las paredes del aula se observa un predominio del abecedario; es un saber considerado necesario y previo a la escritura, utilizado por los estudiantes cuando tienen que hacer ejercicios y para completar palabras. Se muestra como artefacto escolar pensado para la alfabetización de niños y se presenta como algo "dado" y universal, como un modo de encerrar y ordenar el universo de las letras para acercarlas a los aprendices lectores y escritores. Se 
abona así en la escuela la idea de una escritura descontextualizada de otras actividades sociales.

Compartimos a continuación un episodio sobre la selección de remeras y camperas que realizan estudiantes de una escuela primaria de jóvenes y adultos. El objetivo es analizar cómo en situaciones con fines no escolares, los estudiantes recurren al uso del abecedario. ${ }^{7}$

Uno de los días observados en la escuela, se entregó esta ropa que había sido previamente solicitada por las estudiantes. Ellas pidieron camperas talla s. Las camperas les quedaron muy ajustadas y solicitaron a la maestra que reclamara unas nuevas, acordes a sus medidas. Se registró que discutieron sobre las medidas de las camperas, las miraron, midieron las mangas con sus brazos y con su espalda. También revisaron la etiqueta de la campera y comprobaron que decía s, tal como habían solicitado. Además, midieron esta campera con otra que tenían algunas de ellas de años anteriores, para lo cual pusieron una sobre otra. Algunas de esas camperas eran talla M o L, según leyeron en la etiqueta. Asombradas, las alumnas manifestaron que no comprendían por qué ese año habían llegado más pequeñas. La maestra mencionó que solicitaría nuevas camperas y que devolvería estas que pidieron.

La clase continuó, pero dos estudiantes seguían preocupadas por las camperas y parecería que estaban buscando alguna posible respuesta. Al final de esta, cuando estaban merendando, Sandra y Estela (otra de las estudiantes) deletrearon el abecedario. Lo hicieron de manera correcta y volvieron la vista sobre los carteles. Estela, entonces, para ratificar el orden de las letras, le preguntó a la maestra: “¿La s está más atrás en el abecedario?”, a lo que la maestra respondió de manera afirmativa. Estela repitió nuevamente el abecedario en voz baja, miró las camperas y preguntó, “¿pero cómo?” (con un gesto entre

7 A los estudiantes del CEJA, la municipalidad de la ciudad de Córdoba, les ofrece camperas y remeras con la inscripción y el logo de las escuelas municipales para que asistan a la escuela. asombro e interrogación). La maestra se dio cuenta de que estaban asociando el orden del abecedario con el tamaño de la campera. Frente a las dudas de la estudiante, la maestra expuso que las tallas $\mathrm{S}, \mathrm{M}, \mathrm{L}$ correspondían a las denominaciones en inglés: small (pequeño), medium (mediano) y large (grande), y les explicó que "no es por el abecedario [que se eligen] los talles de las camperas". Ante esta explicación de la maestra las estudiantes se rieron y exclamaron: “aahh es que nosotros cuando vamos a comprar nunca habíamos pedido así por letra [risas], imagínate si no [...]. Yo voy mirando así, si me queda o no".

En este episodio se puede evidenciar la pedagogización de la literacidad (Street y Street, 2004), ya que el uso descontextualizado del abecedario orienta una mala elección de la remera y la campera. La ubicación de la letra s casi al final de un conjunto de letras fue interpretada por las estudiantes como la talla más grande. A esta situación, que respondía al plano individual cognitivo, se agregó la ausencia de explicitación de la maestra sobre lo que implicaba la selección de la remera y la campera por la talla correcta. Mostró el carácter estático del abecedario colgado adornando un espacio del aula y la ausencia de relación con las prácticas sociales cotidianas de los estudiantes. Se evidenció además la importancia de hacer explícitas las convenciones del código escrito que se trabajaban en la escuela.

Este modo de entender las prácticas de cultura escrita remite al modelo autónomo (Street, 1984) en el que la letra escrita se presenta de manera neutra frente a los usos de esta fuera de la escuela.

En este sentido, los nel permiten analizar la multiplicidad de prácticas asociadas a la lectura y la escritura y posibilitan dar cuenta de la complejidad de los vínculos alrededor de estas prácticas.

\section{El cuento. Una oportunidad para reconocer relaciones de poder}

En esta sección presentamos un análisis del modo como una maestra de nivel primario de jóvenes 
y adultos de la provincia del Chaco (Argentina) abordó el tratamiento de un cuento con su grupo de estudiantes. Las observaciones se realizaron durante el año 2015 en una institución escolar de la modalidad educación permanente de jóvenes y adultos. Se trataba de un grupo conformado por 19 estudiantes: 12 mujeres y 7 hombres. Los bancos estaban dispuestos uno atrás del otro. En primera fila a la izquierda se ubicaban dos señoras indígenas con sus hijas; detrás de ellas se sentaban los varones (de ese mismo lado) y el resto de las mujeres, atrás y hacia el otro lado.

En el aula se encontraba un pizarrón y a su lado izquierdo se observaban globos de papel colgados con la escritura de las fechas de cumpleaños. Arriba del pizarrón, tal como se describió en el apartado anterior, se leía un cartel con el abecedario y en el resto de las paredes, otros carteles con distintas palabras y sus correspondientes dibujos. También había un afiche con un gusano dibujado que en cada parte tenía una letra. El trato con la maestra es diverso; algunos estudiantes la llamaban "seño", los más jóvenes le decían "señorita" y la trataban de manera informal.

El cuento de la clase observada se denominó "Una piedra en el camino", y trataba de un hombre rico que vivía en un castillo cercano a una aldea y destinaba parte de su fortuna a realizar distintas acciones que él consideraba mejorarían la vida de las personas. Según el relato, el hombre pensaba que la gente pobre de la aldea no era afecta al trabajo y por ello elaboró una estrategia para premiar a la persona que demostrara ser trabajadora y se ocupara del bien de los demás. Fue entonces que colocó una bolsa con monedas de oro debajo de una gran piedra en el medio de un camino muy transitado por la comunidad. Quien quitara la piedra, encontraría la bolsa, y de esta manera se quedaría con el premio.

En el primer momento la maestra pidió a los estudiantes que realizaran una lectura individual en silencio. Luego ella, parada en el frente del aula, comenzó a leer una parte del cuento en voz alta y después iba indicando el turno en que cada uno/a de los/as estudiantes de la clase debía leer un párrafo. Ella decía, "ahora vos", "listo”, y así cada estudiante leyó una parte del cuento hasta llegar al final. Los estudiantes leían con bastante fluidez, la mayoría con voz fuerte; en un caso, una lo hizo tan bajito que casi no se oía. Todos siguieron la lectura con la vista en el texto.

Luego de los turnos de lectura, la maestra les consultó si el cuento les había gustado y, como hablaba de una piedra en el camino, les preguntó, en tono jocoso, si ellos tenían algunas piedras en sus vidas. Todos se rieron y una mujer respondió que su piedra en el camino era su marido, otro dijo que su exmujer. En un ambiente de broma muchos siguieron mencionando otras piedras en sus caminos: "las matemáticas" "los inspectores", pero no profundizaron en ninguna de las "piedras" mencionadas o su significado.

El aula, según Kalman (2003), representa una oportunidad de participación, en referencia a la intervención en actividades sociales, pero también da cuenta de relaciones de poder. "En este sentido su significado abarca tanto las acciones de los sujetos como los vínculos que se entablan entre ellos; la participación se articula con el contexto en una situación específica y, a la vez, en su construcción" (Kalman, 2003, p. 43). El caso analizado muestra una lectura lineal e individual del texto sin demasiadas interacciones entre los estudiantes, en la que se observan los señalamientos de la maestra para designar los turnos de lectura. Ella inició la lectura, indicó los turnos y la extensión de estos.

En este caso también se evidencia cómo las prácticas de cultura escrita se dan en el marco de relaciones de poder. En primer lugar, en la elección del texto para trabajar, ya que fue la maestra quien lo seleccionó de acuerdo, sin participación o consenso con los alumnos. Todas estas cuestiones marcan una posición asimétrica, de poder/saber que detenta la maestra respecto de los estudiantes.

En el diálogo iniciado por la maestra y las interacciones que se produjeron entre los presentes, los 
estudiantes mostraron diversas formas de apropiarse del mismo. Durante los intercambios verbales los participantes fueron construyendo asociaciones entre el contenido del cuento y situaciones de su vida cotidiana, haciendo nuevas interpretaciones y atribuyendo nuevos sentidos al texto. Así las piedras en el camino fueron transformándose en maridos, en las matemáticas y en otros escollos que jóvenes y adultos iban encontrando al comentar el texto.

El soporte del texto fue una fotocopia que la maestra entregó a cada alumno a cambio de unas monedas..Bowman (2008) sostiene que la fotocopia puede convertirse en un "texto nuevo" cuando el maestro "tipea o transcribe en una hoja nueva una consigna o un cuento, sin todos los detalles (escritos, gráficos) que pueden aparecer en el soporte original" (p.63). De este modo la fotocopia entregada por la maestra se constituyó en un "texto nuevo", ya que cumplió con varias de las condiciones que menciona Bowman: se trata de una escritura transcripta por la docente, en la que no figuraba el/la autor/a del cuento ni ninguna otra referencia sobre su origen ni contaba con ilustraciones originales.

La maestra utilizó el cuento como contenido y como recurso para desarrollar la secuencia de actividades como sigue: lectura oral individual y colectiva y comentarios sobre el cuento; escritura en el pizarrón de las actividades planteadas; copia en los cuadernos de las actividades; lectura oral de preguntas y discusión de posibles respuestas; escritura de las respuestas en los cuadernos. Trabajo individual; control de la maestra; lectura oral de las respuestas a las actividades. La lectura individual en silencio fue la primera actividad que la maestra indicó a los estudiantes para que se familiaricen con el texto a modo de práctica, antes de hacerlo en voz alta. Con esta actividad, ella aseguró haberse dirigido a fortalecer además aspectos actitudinales porque, sostuvo, "son demasiado tímidos, muy inseguros [...] les cuesta mucho largarse a leer, entonces esa primera mirada les permite tener un pantallazo general de lo que trata la lectura y así luego se animan a hacerlo en voz alta" (Sara, Resistencia, 19 de noviembre, 2016). Según la maestra, la práctica de la lectura es un aspecto muy importante y considera que debe ejercitarse para llegar a dominarla. Por ello en la clase también les demandó — además de la lectura silenciosa - leer en voz alta algunos párrafos por turnos, comenzando con ella e indicando luego a otros el orden de lectura y la extensión. Para justificar esta actividad expresó: "no leen solos, no se animan. En muy contados casos se animan a leer solos, sin que les pidas" (Sara, Resistencia, 19 de noviembre, 2016).

Esto es congruente con la observación de Kalman (2004), en una experiencia de lectoescritura con un grupo de mujeres de Mixquic (México). Ella identificó que al inicio del proyecto estas mujeres "insistían en su desconocimiento de la lectura y la escritura, dudaban de su capacidad intelectual, devaluaban su experiencia y conocimientos" (p. 112).

De igual modo, la maestra de este estudio, sostenía que inicialmente se mostraban muy inseguros/ as frente a la posibilidad de aprender a leer y escribir mejor o de acceder a nuevos contenidos. Sin embargo, la lectura oral en ese grupo propició “[...] el desarrollo de modalidades solidarias para leer juntas; se ayudaban entre sí para descifrar palabras desconocidas o comentaban algunos aspectos del lenguaje $[. .] ”.($ p. 118).

Al analizar la participación de los estudiantes en el intercambio de la clase observada, se identificó que los más participativos fueron los/as de mayor edad, el resto simplemente se mantuvo escuchando. Respecto de los intercambios que puede provocar la lectura compartida cuando es significativa para las personas, Kalman (2004) describió los efectos que produjo en el grupo de mujeres (mencionado anteriormente) la lectura de la autobiografía de una luchadora social mexicana, especialmente su pelea personal por superarse y vivir la vida según sus principios. La experiencia de esta mujer y el acercamiento a la de estas mujeres hizo que el grupo se identificara con ella y que se 
entablaran "importantes discusiones éticas, morales y políticas acerca de temas importantes para ellas [...] como una forma de comprender el texto y sus propias experiencias como madres, abuelas y mujeres $[\ldots] ”($ p. 114).

Al comparar la experiencia de lectura en clase que aquí se presenta y la experiencia relatada por Kalman, se evidenció la importancia de la selección del material de lectura, ya que, cuando resulta significativo para la vida de las personas, profundiza las posibilidades de comprensión no solo del texto, sino también de la propia vida o de su entorno. De otro modo, la lectura del texto solo promueve un intercambio de ideas que quedan en el plano de la superficialidad. El principal objetivo de la maestra no fue el desarrollo de una estrategia para provocar un pensamiento reflexivo, sino que se sintieran fortalecidos y mas seguros a través de su práctica lectora. Ella consideró que era necesario que los jóvenes y adultos puedan expresar sus sentimientos, construir un grado de confianza entre compañeros y con la maestra para facilitar los entornos de trabajo. Con ello intentó crear un ambiente cordial, de compañerismo, de solidaridad, de ayuda mutua, en el que pudieran generarse mejores condiciones para el aprendizaje. Al respecto expresó “... el hecho de compartir también [...] lo que quiero es que se ayuden mutuamente entre ellos, pero que no le haga el uno al otro la tarea” (Sara, Resistencia, 19de noviembre, 2016).

En el pizarrón, la maestra consignó la fecha del día, el nombre del cuento y la actividad que se iba a realizar, a saber: “¿quiénes son los personajes del cuento?, ¿cuál es el problema que se presenta?, ¿cómo se resuelve?, cambia el título del cuento, escribe una reflexión".

Si bien esta actividad era muy similar a la que realizan los maestros en las escuelas primarias para niños, la maestra comentó que esta le permitió: "ver la comprensión del tema, cosas que no entienden ellos", pero también facilitarla, "que puedan relacionar, que puedan llegar a una comprensión del texto" (Sara, Resistencia, 19 de noviembre, 2016).
Sin embargo, hablando de la comprensión de lectura, Rockwell (1985) sostiene que no es una habilidad abstracta y que los conocimientos nunca se extraen de una manera simple del texto escrito. Para esta autora es necesario llevar conocimientos al texto para poder comprenderlo, estableciendo una relación "independiente con el fenómeno expresado en el texto o bien una relación social, mediadora, en el proceso de apropiación de conocimientos a partir de la lectura" (p.303), cuestiones que no se evidenciaron en este caso.

Una vez que la maestra terminó de copiar las actividades en el pizarrón, pidió a los estudiantes que realizaran una copia de ellas en sus cuadernos. Posteriormente leyó en voz alta las preguntas y entre todos/as las iban contestando oralmente - algunas la maestra y otras los estudiantes-. Cuando se compartía la respuesta de cada pregunta en voz alta, la maestra solicitaba a los estudiantes que la escribieran en sus cuadernos. Lo hicieron individualmente, en silencio absoluto. Por el contrario, en la experiencia relatada por Kalman (2004) la lectura oral en ese grupo propició “[...] el desarrollo de modalidades solidarias para leer juntas; se ayudaban entre sí para descifrar palabras desconocidas o comentaban algunos aspectos del lenguaje [...]” (p. 118).

Finalmente, volvieron a leer las preguntas y las respuestas en voz alta en los turnos que dictó la maestra, mientras cotejaban las respuestas con las escritas en sus cuadernos. Como lo hizo en otras actividades, la maestra controló pasando por los bancos. Pero según sus decires, el control no es la única intención, sino que a través de las actividades propuestas, buscaba generar lazos en el aula. De este modo, volvió a inscribir las estrategias en aspectos actitudinales.

Los estudiantes escribieron "ser bueno tiene recompensa $[. .$.$] hacer el bien te cambia la vida”.$ Observamos que las reflexiones finales que los estudiantes expresaron sobre el cuento están en consonancia con una moraleja: "haz el bien y serás recompensado”. Estas prácticas se sostienen a lo 
largo de la historia y muestran cómo la escuela como institución pública recurrió a fundamentos morales y religiosos, mediante los cuales controlar a las clases populares. La identificación entre lectura y moralidad se convierte así en una de las características de la escritura escolar (Kalman, 2017).

Si bien el recurso de la literatura resulta muy oportuno para que jóvenes y adultos puedan apropiarse de prácticas de lectura y escritura y acceder a nuevos contenidos, sería más enriquecedor si el cuento no hubiera sido seleccionado de la literatura infantil (La piedra en el camino, s. f.). Incluso, en este cuento en particular advertimos un mensaje estigmatizador en la generalización entre personas pobres y su falta de afección al trabajo.

En las indagaciones realizadas, este cuento también aparece asociado a páginas religiosas con mensajes morales. La naturaleza del relato y las intervenciones de la maestra no permitieron contextualizarlo y, por lo tanto, impidieron la discusión de otros aspectos más amplios en torno a la situación descripta. Por esta razón, no permitió a los/as estudiantes vincular el contenido con problemáticas de su vida cotidiana salvo tangencial y superficialmente, como se mencionó arriba.

De todos modos, en esta investigación se sostiene que, independientemente de los contenidos trabajados, es la intervención de la maestra a partir de las actividades y textos propuestos la que permite o no que los estudiantes se apropien de ciertos contenidos. Si este mismo cuento hubiera sido contextualizado en el tiempo y se hubiera presentado la biografía de su autor, habría brindado otra perspectiva desde la cual analizar aspectos como: las desigualdades sociales, la estigmatización de los grupos vulnerables, la caridad, entre otros.

\section{Palabras para cerrar}

Los estudios que compartimos en este trabajo tienen como eje común problematizar las prácticas de lectura y escritura en escuelas de jóvenes y adultos. De este modo, nuestra mirada se focaliza en los sujetos de estas escuelas y en su relación con la cultura escrita. Para hacerlo, presentamos dos investigaciones que analizamos a la luz de cómo se configuran las prácticas de lectura y escritura en el espacio escolar, considerado históricamente como el ámbito privilegiado para apropiarse de la cultura escrita.

Es necesario recuperar la perspectiva de los nel para desagregar tres grandes aportes de los estudios presentados. En primer lugar, permite comprender que se trata de prácticas sociales, históricas, situadas y, como tales, insertas en relaciones de poder. En segundo lugar, esta visión da cuenta de los usos sociales de la lengua escrita, más allá de la posibilidad de leer y escribir autónomamente. $Y$, en tercer lugar, propicia el reconocimiento de los saberes que los sujetos tienen sobre la cultura escrita, sus usos y sus consecuencias sociales. Estos aspectos son fundacionales y constituyen un abanico de miradas sobre el acceso a la cultura escrita en el campo de la educación de jóvenes y adultos.

Por otra parte, entender la lectura y la escritura como prácticas sociales propicia el estudio sobre los recursos materiales y simbólicos existentes de la cultura escrita, así como también sobre las percepciones de jóvenes y adultos en la escuela acerca de la cultura escrita y de sí mismos como lectores y escritores.

Estos modos de analizar las prácticas de literacidad ofrecen herramientas para superar la mirada dicotómica acerca de los sujetos que leen o no leen, que escriben o no escriben. En este sentido, señalamos la posibilidad de comprender que existen grados de literacidad que permiten identificar objetos escritos a través de diversas huellas o registros, que se pueden conocer algunas letras y que se lee la realidad desde otros modos. Ya no se trata de "analfabetos" que no pudieron o no quisieron acceder a la escolaridad y llevarla a buen término, sino de personas con conocimientos y saberes acerca de la lengua escrita, que arrastran consigo historias de discriminaciones y desvalorizaciones por no haber transitado por el sistema educativo. 
En los espacios formativos de educación de jóvenes y adultos, en pocas ocasiones se considera la perspectiva de los nel. Los futuros maestros de esta modalidad están formados para trabajar con niños, y no se contempla la posibilidad de ejercer la profesión con jóvenes y adultos en las distintas materias o asignaturas. De este modo, en su gran mayoría, los docentes hacen uso de los mismos modos y artefactos utilizados para la enseñanza de niños, tal como aquí lo evidenciamos. Esto se cuestiona como "infantilización de la práctica en adultos", ante lo que cabe tener en cuenta que muy pocas veces se observan procesos de formación docente específica.

En nuestra experiencia de trabajo con maestros registramos una constante preocupación sobre las maneras de enseñar a leer y escribir, pero en distintas ocasiones señalamos la ausencia de conocimientos sobre los reales saberes y conocimientos que tienen los jóvenes y adultos. Pareciera que es cosa juzgada el hecho de encontrar a sujetos que vienen con experiencias diversas. Sin embargo, pocas veces observamos prácticas docentes que entablen un diálogo con esos conocimientos y puedan desde allí configurar procesos de enseñanza y aprendizaje.

Más bien encontramos prácticas que se basan en la copia del pizarrón, el corte de textos de revistas y, allí, la identificación de sílabas y palabras. Muchas veces estamos frente a actividades que no tienen significación alguna para la vida de los adultos. Sobre todo, cuando podemos reconocer la variedad de usos sociales de la lengua escrita por fuera del espacio escolar.

En el caso del uso del abecedario se observa el tratamiento "neutral", sin más explicaciones de la maestra sobre lo que significa una letra en relación con una talla de ropa. Se evidencia la hipótesis que formulan los estudiantes sobre el lugar que ocupa la letra en el abecedario y el tamaño del objeto. Cuanto más atrás se encuentre la letra el objeto más grande debe ser. Esta asociación es solo un modo de mostrar las consecuencias de un tratamiento descontextualizado del abecedario, como codificación de fonema en grafema sin medir las consecuencias sociales de este conocimiento, lo que revela la ausencia del uso social de la lengua escrita. Esta situación anula la posibilidad de dar cuenta de las múltiples significaciones y usos que hacen de la lengua escrita los jóvenes y adultos.

En el otro caso presentado, el análisis del cuento muestra la selección de un texto literario que estigmatiza a sectores sociales económicamente desfavorecidos bajo la presunción de que se trata de una población que no es afecta al trabajo, lo que pone en evidencia un sesgo moral del texto. Ante esto nos preguntamos: ¿Cómo se refleja la vida cotidiana de los estudiantes en relación con sus trabajos o con su realidad de desempleados? ¿Cómo se contextualizan las piedras que cada quien encuentra en su camino de la vida? Ellos comparten sus "piedras", pero la maestra no lo hace de la misma manera.

El uso de la literatura en la escuela muestra una decisión acertada; los textos literarios pueden promover el disfrute de la lectura, permiten entablar la comunicación con uno mismo y con otros, potencian procesos de pensamiento, despiertan la imaginación, la curiosidad, y permiten mirar y conocer el mundo desde otra perspectiva. Estas razones fundamentan la necesidad de considerar su uso al momento de construir procesos con jóvenes y adultos, cuya trayectoria social está plagada de exclusiones y carencias, entre ellas, el acceso a ciertos bienes culturales. Un ejemplo de cómo usar la literatura para enriquecer la experiencia de la alfabetización es la experiencia narrada de Kalman (2004) con mujeres de Mixquic, México, donde se demostró que cuando los textos seleccionados brindan a los sujetos la posibilidad de vincularlos con sus experiencias vitales dan lugar reflexiones de profundo significado para analizar posiciones y decisiones personales de esos sujetos.

El potencial de la perspectiva teórico-metodológica de los NEL en el campo de la educación de 
jóvenes y adultos permite identificar el abordaje de la literacidad en el aula y reconocer los conocimientos circulantes y las prácticas de cultura escrita de sujetos jóvenes y adultos que no se "adecuan" a los cánones escolares. Este reconocimiento nos aleja del lugar de infante asignado históricamente a los jóvenes y adultos en las aulas y brinda trazas para repensar los procesos de formación y prácticas de los docentes de la modalidad.

\section{Referencias}

Arrieta, R. (en preparación). La escolarización y sus consecuencias sociales. Un análisis de los usos sociales del conocimiento escolar en jóvenes y adultos de la ciudad de Córdoba. Centro de Investigaciones Facultad de Filosofía y Humanidades. Córdoba: UNC.

Arrieta, R. y Lorenzatti, M. del C. (2017). La escuela de jóvenes y adultos un espacio para aprender 'la charla' y la 'buena escritura'. En A. F. Ribeiros de Freitas y M. L. de Queiroz Freitas (Eds.), Os sentidos da formação em rede (pp. 139-151). Maceio, Alagoas, Brasil: Edufal.

Blazich, G. (en preparación). Itinerarios. Ser maestra en educación de jóvenes y adultos. Centro de Investigaciones Facultad de Filosofía y Humanidades. Córdoba: UNC.

Bowman, A. (2008). El maestro como mediador en la construcción de la lengua escrita. Trabajo final de licenciatura en Ciencias de la Educación (no publicado). Universidad Nacional de Córdoba, Córdoba, Argentina.

Cook-Gumperz, J. (1988). La construcción social de la alfabetización. España: Paidós.

Ferreiro, E., y Teberosky, A. (1980). Los sistemas de escritura en el desarrollo del niño. México: Siglo xxi.

Freire, P. (1971). La educación como práctica de la libertad. Buenos Aires: Siglo XXI.

Heller, A. (1977). Sociología de la vida cotidiana. Barcelona, España: Península.

Kalman, J. (2002). La importancia del contexto en la alfabetización. Revista Interamericana de Educación de Adultos, 24(3), 11-27. Pátzcuaro: CREFAL.

Kalman, J. (2003). Escribir en la plaza. México: Fondo de Cultura Económica.
Kalman, J. (2004). Saber lo que es la letra. Una experiencia de la lectoescritura con las mujeres de Mixquic. México: Siglo XXI.

Kalman, J. (2008). Beyond definition: Central concepts for understanding literacy. International Review of Education, 54(5), 523-538.

Kalman, J. (2015). El origen social de la palabra propia. En J. Kalman y M. E. López (Eds.), Palabras que zurcen. México: Conaculta.

Kalman, J. (2017). Por qué ma me mi mo mu sigue en la escuela. En A. F. Ribeiros de Freitas, M. L. de Queiroz Freitas (Eds.), Os sentidos da formação em rede (pp. 189-207). Maceió, Alagoas: Edufal.

Kalman J. y Street, B. (coordinadores) (2009). Lectura, escritura y matemáticas como prácticas sociales. Diálogos con América Latina. México: CREFAL-Siglo XXI.

Knobel, M. y Kalman, J. (2017). Aprendizaje docente y nuevas prácticas de lenguaje. Posibilidades de formación en el giro digital. Biblioteca Innovación Educativa. México: sM de Ediciones.

La piedra en el camino (s. f.). Cuentos cortos. Disponible en: https://www.encuentos.com/cuentos-cortos/ la-piedra-en-el-camino-cuentos-para-pensar-cuentosbreves-reflexiones/

Rockwell, E. (1985). Ser maestro, estudios sobre el trabajo docente. México: Caballito.

Rockwell, E. (1997). De huellas, bardas y veredas: una historia cotidiana en la escuela. En E. Rockwell (Coord.), La escuela cotidiana (pp. 13-57). México, D. F.: Fondo de Cultura Económica.

Rockwell, E. (2005). La apropiación, un proceso entre muchos que ocurren en ámbitos escolares. En Memoria, conocimiento y utopia (pp. 28-38). Anuario somehide. Barcelona-México: Pomares, s. A.

Rockwell, E. (2009). La experiencia etnográfica. Historia y cultura en los procesos educativos. Buenos Aires: Paidós.

Sanjuán, M. (2015). Los abecedarios ilustrados como "artefactos" estéticos y literarios: aproximación a su poética. Ocnos, Revista de Estudios sobre lectura, 14, 42-64. DOI: 10.18239/ocnos_2015.14.04

Street, B. V. (1984). Literacy in theory and practice. Cambridge: Cambridge University Press.

Street, B. V. (1993). Cross-cultural approaches to literacy. Nueva York: Cambridge University Press. 
Street, J. C. y Street, B. V. (2004). La escolarización de la literacidad. En V. Zavala, M. Niño Murcia y P. Ames (Eds.), Escritura y sociedad. Nuevas perspectivas teóricas y etnográficas (pp. 181-210). Lima: Red para el Desarrollo de las Ciencias Sociales en el Perú.

Street, B. (2008). Nuevas alfabetizaciones, nuevos tiempos ¿Cómo describimos y enseñamos los conocimientos, las habilidades y los valores acordes con las formas de alfabetización que las personas necesitan para los nuevos tiempos? Revista Interamericana de Educación de Adultos, 30(2), 41-69. Pátzcuaro: CREFAL.

Tfouni Verdiani, L. (2010). Letramento e alfabetização (9. Ed.). São Paulo: Cortez.
Zavala, V. (2002). (Des) encuentros con la escritura: escuela y comunidad en los Andes peruanos. Lima: Red para el Desarrollo de las Ciencias Sociales en el Perú.

Zavala, V. (2004). Literacidad y desarrollo. Los discursos del Programa Nacional de Alfabetización en Perú. En V. Zavala, M. Niño Murcia y P. Ames (Eds.), Escritura y sociedad. Nuevas perspectivas teóricas y etnográficas (pp. 437-459) Lima, Perú: Red para el Desarrollo de las Ciencias Sociales en el Perú.

Zavala, V., Murcia, M. y Ames, P. (Eds.). (2004). Escritura y sociedad. Nuevas perspectivas teóricas y etnográficas. Lima, Perú: Red para el Desarrollo de las Ciencias Sociales en el Perú.

How to reference this article: Lorenzatti, M. C., Blazich, G. y Arrieta, R. (2019). Aportes de los Nuevos Estudios de Literacidad al campo de la educación de jóvenes y adultos. Íkala, Revista de Lenguaje y Cultura, 24(2), 291-305. Dor: 10.17533/udea.ikala.v24n02a06 